\title{
An Experimental Test of the Scaling Prediction for the Spatial Distribution of Water during the Drying of Colloidal Films
}

\author{
P. Ekanayake ${ }^{1}$, P.J. McDonald ${ }^{2}$, and J.L. Keddie, ${ }^{\text {a2 }}$ \\ 1 Department of Physics, University of Peradeniya, Peradeniya, Sri Lanka \\ 2 Department of Physics, University of Surrey, Guildford GU2 7XH, UK
}

\begin{abstract}
A Peclet number, $P e$, for the drying of colloidal films can be used as a predictor of the uniformity of water concentration in the direction normal to the film. Uniform drying is predicted to occur when $P e<1$, whereas with $P e>1$, a layer of packed particles is expected to develop above a more dilute layer. Routh and Zimmerman have more recently proposed that the particle concentration gradient between the packed and dilute layers, $d \phi_{p} / d z$, will scale as $P e^{1 / 2}$. Here, this scaling relation is tested experimentally with magnetic resonance profiling data obtained from waterborne colloidal films dried under conditions to yield a range of $P e$. It is found that $d \phi_{p} / d z$ increases with $P e$ but scales as $P e^{0.8}$. This disagreement with the prediction can be attributed to an underestimate of $P e$ when there are greater non-uniformities of drying, because of an unquantified slowing down of the evaporation rate.
\end{abstract}

\section{Introduction}

Waterborne colloidal polymer films are being used increasingly more often in applications, such as adhesives and coatings, which previously relied on casting polymers from organic solvents [1]. Issues relating to drying are consequently growing in interest and are motivating scientific study [2]. When drying ovens are used in a production process, the drying rate is naturally very fast, but there is an increased risk of residual water entrapment and the potential for an accompanying detrimental effect on mechanical, [3] visual, and adhesive [4] properties. In other applications, such as architectural coatings, the time during which a wet colloidal film can be smoothed or re-worked without leaving a permanent impression, known as the open time, is of particular interest [5]. There is often a need in waterborne coatings to increase the open time. Ways to ensure a uniform distribution of particles in the film so as to keep the viscosity from diverging at high solids content are therefore needed.

Questions about the distribution of colloidal particles in drying polymer films have inspired many researchers to carry out modelling [6-8] and experimentation [9-12] in recent years. Some research has considered the drying process laterally in the film (i.e. parallel to the substrate) $[13,14]$. Drying in the direction perpendicular to the substrate (i.e. vertically), however, is of greater relevance to problems associated with short open times and skin formation [15], which is when a coalesced layer develops at the surface of a drying film. With skin formation, the rate of drying is thereafter slower [16], and there is a risk of water entrapment within the film or in the substrate. The vertical water distribution during drying also influences the distribution of surfactants within the dry film [17].

\footnotetext{
a e-mail: j.keddie@surrey.ac.uk
} 

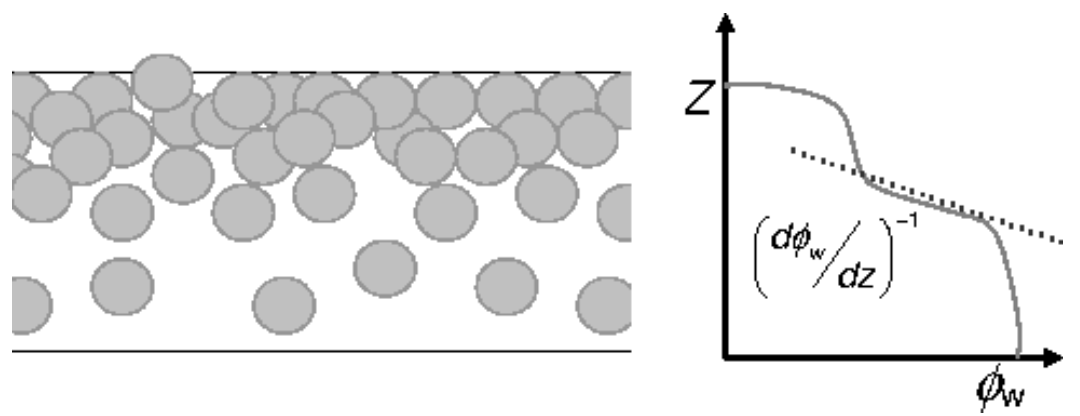

Fig. 1. (left) Schematic diagram of non-uniform drying in the vertical direction within a waterborne colloidal film and (right) the corresponding water concentration profile showing the water concentration gradient between the packed region at the top and the underlying dilute region.

A prevailing model of film formation, developed by Routh and Russel (R-R) [18], predicts that there will be skin formation, in those circumstances when there is non-uniform vertical drying combined with wet sintering, which is particle deformation driven by a reduction in the polymer/water interfacial energy. In the R-R model of drying $[6,13,18]$, a Peclet number for drying, $P e$, is used to indicate the relative contributions of water evaporation and Brownian diffusion of the dispersed particles in determining the concentration profile in the vertical direction. The evaporation rate, $E$, is expressed as the velocity of the recession of the water surface. For a film of initial thickness, $H$, the characteristic time to dry, $\tau_{d r y}$, is then expressed as $\sim H / E$. The characteristic time for a Brownian particle to diffuse from top to bottom in the film is given as $\tau_{\text {diff }} \sim H^{2} / D_{S E}$, where $D_{S E}$ is the Stokes-Einstein diffusion coefficient. The ratio of these two characteristic times is used to define $P e$, such that $P e=\frac{\tau_{d i f f}}{\tau_{d r y}} \sim \frac{H E}{D_{S E}}$.

For particles of radius $R$ dispersed in a fluid with a viscosity of $\mu, P e$ can be expressed as $P e=\frac{6 \pi \mu R H E}{k T}$ from the definition of $D_{S E}$ in the dilute limit, where $k$ is the Boltzmann constant, and $T$ is the absolute temperature. When $P e \ll 1$, the particle volume fraction, $\phi_{p}$, in the film is predicted to be uniform across the film depth, because the time for diffusion to re-distribute the particles accumulating near the film surface is short relative to the drying time. While with $P e$ $\gg 1$, a gradient in the water concentration is predicted, with less water near the interface with air (as illustrated in Figure 1). A packed particle layer, with a volume fraction, $\phi_{m}$, is predicted to accumulate above a dilute layer. The existence of packed particle layers at the surface of drying latex films has been vividly confirmed to exist in the electron micrographs of Ma et al. [19] obtained from cross-sections of cryogenically-fractured films. Gorce et al. [20] have used magnetic resonance (MR) profiling to determine the vertical water concentration profile, $\phi_{w}(z)$, in drying colloidal films. They observed greater non-uniformity in $\phi_{w}(z)$ as $P e$ was increased, in general agreement with the concepts of the R-R model. Analogous models have been developed for the solvent distribution in films cast from polymer solutions [21,22]. More recently, Routh and Zimmerman (R-Z) [23] have looked more closely at the interface between wetter and drier regions of non-uniformly drying colloidal films. After performing numerical simulations and an asymptotic analysis, they went on to derive a scaling law for the particle concentration gradient $\mathrm{d} \phi_{p} / \mathrm{d} z$ in the boundary region between the packed layer (with $\phi_{p} \sim \phi_{m}$ ) lying above the dilute layer (with $\phi_{p} \sim \phi_{0}$, the initial concentration). At higher values of $P e$, when particle packing at the film surface is predicted, $d \phi_{p} / d z$ is predicted to vary as $P e^{1 / 2}$. The objective of this work is to test this scaling prediction experimentally, using magnetic resonance profiling in a GARField magnet [24]. This technique determines $\phi_{w}(z)$. However, assuming there are no air voids, then $\phi_{w}+\phi_{p}=1$, and the two gradients are equal in magnitude but merely opposite in sign. This work is motivated by a practical need to achieve uniform drying in waterborne coatings without the formation of a skin layer. The work will reveal the extent to which a knowledge of $P e$ for a drying film can be used as a quantitative predictor of drying non-uniformity. 


\section{Experimental Details}

\subsection{Materials}

An acrylic latex was prepared by emulsion polymerization. A copolymer was polymerized from butyl acrylate and methyl methacrylate in a 1:1 weight ratio, using an anionic surfactant (Dowfax 2A1). A mixture of ammonium persulfate and MBS was used as the initiator. The average particle diameter, according to dynamic light scattering measurements, was $2 R=180 \mathrm{~nm}$ and the solids content was 50 wt.\%. The continuous phase was assumed to be pure water, and its viscosity [25] was taken to be $\mu=1.0 \times 10^{-3} \mathrm{~Pa}-\mathrm{s}$ in calculations of $P e$. The glass transition temperature, $T_{g}$ of the copolymer, as obtained from dried films via differential scanning calorimetry, was $18^{\circ} \mathrm{C}$. The experiments were performed at a temperature of $22^{\circ} \mathrm{C}$, which is just $4^{\circ} \mathrm{C}$ above the polymer's $T_{g}$.

\subsection{Procedure}

Measurements of $\phi_{w}(z)$ in the drying acrylic latex films were obtained using magnetic resonance ${ }^{1} H$ profiling with a GARField magnet, described elsewhere [15,20,24]. Wet films were cast onto clean glass coverslips $(2 \mathrm{~cm} \times 2 \mathrm{~cm})$ and immediately placed in the magnet at a position corresponding to a magnetic field strength of $0.7 \mathrm{~T}$ and a field gradient strength of $17.5 \mathrm{~T} \mathrm{~m}^{-1}$. Signals were obtained using a quadrature echo sequence [26]: $90_{x}-\tau-\left(90_{y}-\tau-e c h o-\tau-\right)_{n}$ for $n=32$ echoes and a pulse gap of $\tau=95.0 \mu \mathrm{s}$. To obtain a profile, the echoes were Fouriertransformed and then summed, thus giving an NMR signal intensity profile as a function of vertical position. No significant signal is observed from the polymer phase, and the water spinspin relaxation time is largely independent of the polymer concentration in the dispersion. Hence, the integrated NMR intensity is a fair measure of the water content, and it can be calibrated from the time-zero data with a known, uniform concentration. The pixel resolution in these experiments was about $10 \mu \mathrm{m}$. To correct for the sensitivity decline over the film thickness, profile intensities were normalized by an elastomer standard. Films were dried under a range of conditions. To obtain high $P e$ values, the flow rate of air across the film surface, and hence $E$, was increased. Low $P e$ values were obtained in still air and by raising the local humidity in an enclosure.

\section{Results}

As film formation occurred just $4^{\circ} \mathrm{C}$ above the copolymer's $T_{g}$, the particle should be highly viscous under the experimental conditions, and particle deformation will be slow. In this case, particle deformation is expected to result mainly from dry sintering $[6,18]$. Atomic force microscopy of dry films found that particle identity is indeed retained at the surface of freshly-dried films. Hence, it is justified to consider the particles as spheres during the water evaporation stage, as assumed in the R-R Peclet description, as deformation is not expected until the later stages of film formation.

To ensure a fair comparison between MR profiles, time is expressed as a normalized time. The normalised time is found from the time after film casting divided by $\tau_{d r y}$. For this calculation, $E$ is determined experimentally from the wet film thickness found in the first several MR profiles, such as demonstrated in Figure 2. Under still air with a relative humidity of $53 \%$, $E$ is $7.1 \times 10^{-8} \mathrm{~ms}^{-1}$. The evaporation rate was varied in the range from $1.25 \times 10^{-9} \mathrm{~ms}^{-1}$ to $2.50 \times 10^{-7} \mathrm{~ms}^{-1}$ through control of the environmental conditions.

Figure 3 compares two MR profiles obtained from drying films having different $P e$ values. Both profiles were obtained at approximately the same normalized time of 0.2 . As noted previously, the NMR intensity on the vertical axis is proportional to $\phi_{w}$. The intensity profile has been divided by the first profile (obtained at a time of 0 ), such that the range of intensity will lie between 0 and 1 . The intensity of 1 corresponds to the water volume fraction in the initial latex $\left(\phi_{w} \sim 0.5\right)$. In each profile, it is seen that the water concentration is lower near the upper 


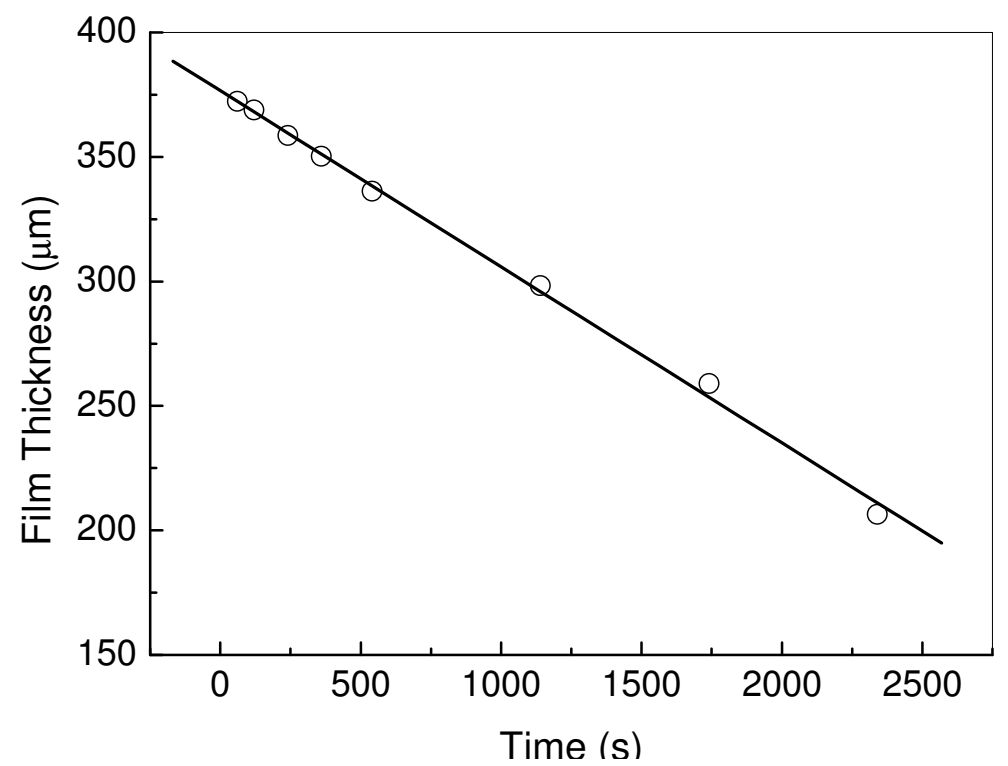

Fig. 2. Decrease of the thickness of a wet latex film with time in still air at a temperature of $20{ }^{\circ} \mathrm{C}$ and relative humidity of $53 \% \mathrm{RH}$. The best-fit line corresponds to $E=7.1( \pm 0.1) \times 10^{-8} \mathrm{~m} / \mathrm{s}$.

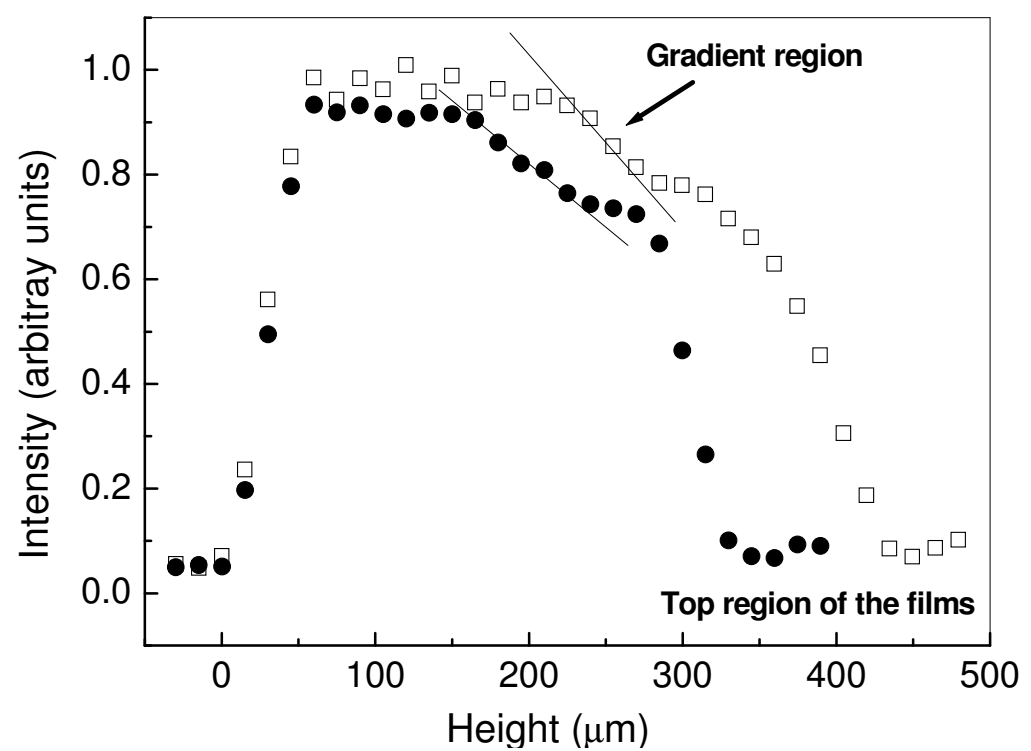

Fig. 3. GARField MR profiles of two latex films dried under conditions corresponding to $P e=13$ (open circles) and $P e=49$ (filled squares). The signal intensity is proportional to $\phi_{w}$.

film surface, represented toward the right-side of the profile. There is an apparent water concentration gradient near the middle of each film, separating a packed layer on the upper side and a more dilute region below. In the MR profile obtained from a drying film with $P e=49$, the concentration gradient appears to be stronger in comparison to the profile obtained with a lower $P e(13)$. This result is in general agreement with the model predictions.

The apparent slope in the MR profiles in Figure 3 is less than the true instantaneous $d \phi_{w} / d z$ because of two factors. First, there is profile broadening due the inherent point-spread function of the experiment, including imperfect alignment of the sample plane with respect to the mag- 
netic field gradient direction. A second factor is a smearing of the profile because of the linear (with time) recession of the wet surface with water evaporation during the finite measurement time. Quantitative analysis of the concentration gradients requires that these factors are accounted for. Because a method for the correction of the concentration gradients has not been previously reported for the MR profiling of colloidal films, we briefly discuss our approach here.

An estimate of the broadening caused by the point-spread function (psf) can be made through examination of the profile of the interface between the wet film and the non-permeable glass substrate. This broadening is usually over two or three pixels, but it is less than that caused by the surface recession. De-convolution of the psf broadening is a notoriously difficult procedure, and no attempt was made to correct for it.

A simple geometric argument has been used to correct for the smearing from surface recession during the measurement time. An analysis of the slope of the profile near the upper film surface was applied, as follows. Figure 4a shows a schematic diagram of a drying profile. Here, $S_{\text {obs }}$ represents the slope that is observed in an MR profile between the dilute and packed regions, whereas $S_{a c t}$ represents the actual (true) slope without the smearing. The slope in the profile at the boundary between the wet surface and the atmosphere is represented as $S_{d r y}$. In all cases, $S_{d r y}>S_{o b s}$, because $S_{d r y}$ represents the broadening caused by the recession, whereas $S_{\text {obs }}$ is affected by the combined effects of this broadening plus the particle concentration gradient in the boundary region. A geometric construction of the slopes is presented in Figure $4 \mathrm{~b}$. It is demonstrated in the construction how $S_{a c t}$ can be calculated from measurements of $S_{d r y}$ and $S_{o b s}$ and the application of trigonometry.

This correction was adopted in the analysis of the MR profiles obtained from a series of latex films corresponding to a range of $P e$ values. The water concentration gradient, $\mathrm{d} \phi_{w} / \mathrm{d} z$, was obtained from the corrected profiles at the boundary between the dilute and packed regions. The R-Z simulations [23] indicated that 0.2 is an appropriate normalized time to test the R-Z scaling predictions, and so it was chosen for analysis. For consistency in the analysis, four pixels in the center of the gradient region were selected. Then the best, linear fit was obtained from a linear regression analysis. The error on the measurement was obtained by shifting the range of data points up or down by one point.

The results of the analysis over a range of $P e$ are shown in Figure 5 . There is a clear increase in $\mathrm{d} \phi_{w} / \mathrm{d} z$ with $P e$. On these logarithmic axes, $\mathrm{d} \phi_{w} / \mathrm{d} z$ is seen to vary with $P e$ with a slope of $0.8 \pm 0.2$, indicating that $\mathrm{d} \phi_{w} / \mathrm{d} z \sim P e^{0.8}$. Even within the uncertainty of the measurement, these experimental results do not support the R-Z scaling prediction of $d \phi_{w} / d z \sim P e^{0.5}$.

This difference between our experimental results and the scaling predictions may be due to either an increasing overestimate of $\mathrm{d} \phi_{w} / \mathrm{d} z$ with increasing $P e$ or alternatively an increasing underestimate of $P e$ with increasing $\mathrm{d} \phi_{w} / d z$. Although there could be a systematic error in $\mathrm{d} \phi_{w} / d z$ leading to a shift of the data, it is not obvious why this error would increase with $P e$. On the other hand, there are several factors that influence the calculation of $P e$. The main parameter that was used to adjust $P e$ experimentally was $E$, and $E$ was calculated from the rate of decrease in $H$ during the first several minutes of the experiment. In those experiments in which $E$ is high, there could have been particle accumulation at the film surface from an early stage in the drying process, thus slowing down the rate of water loss. Hence, in experiments with higher $P e$, the calculation of $P e$ could have used a value of $E$ that was lower than the free value of evaporation without the effects of particle accumulation. This is one possible reason why $P e$ might have been under-estimated in experiments in which there is a larger $\mathrm{d} \phi_{w} / \mathrm{d} z$. The effect is gradual, however, so that a linear relationship is still found on a plot on log-log axes.

\section{Conclusions}

The water concentration gradient in drying colloidal films was found to scale as $\sim P e^{0.8}$, which is a stronger dependence than the R-Z prediction of $d \phi_{p} / d z \sim P e^{1 / 2}$. This discrepancy could be attributed to the effects of skin formation at faster evaporation rates leading to an underestimate 

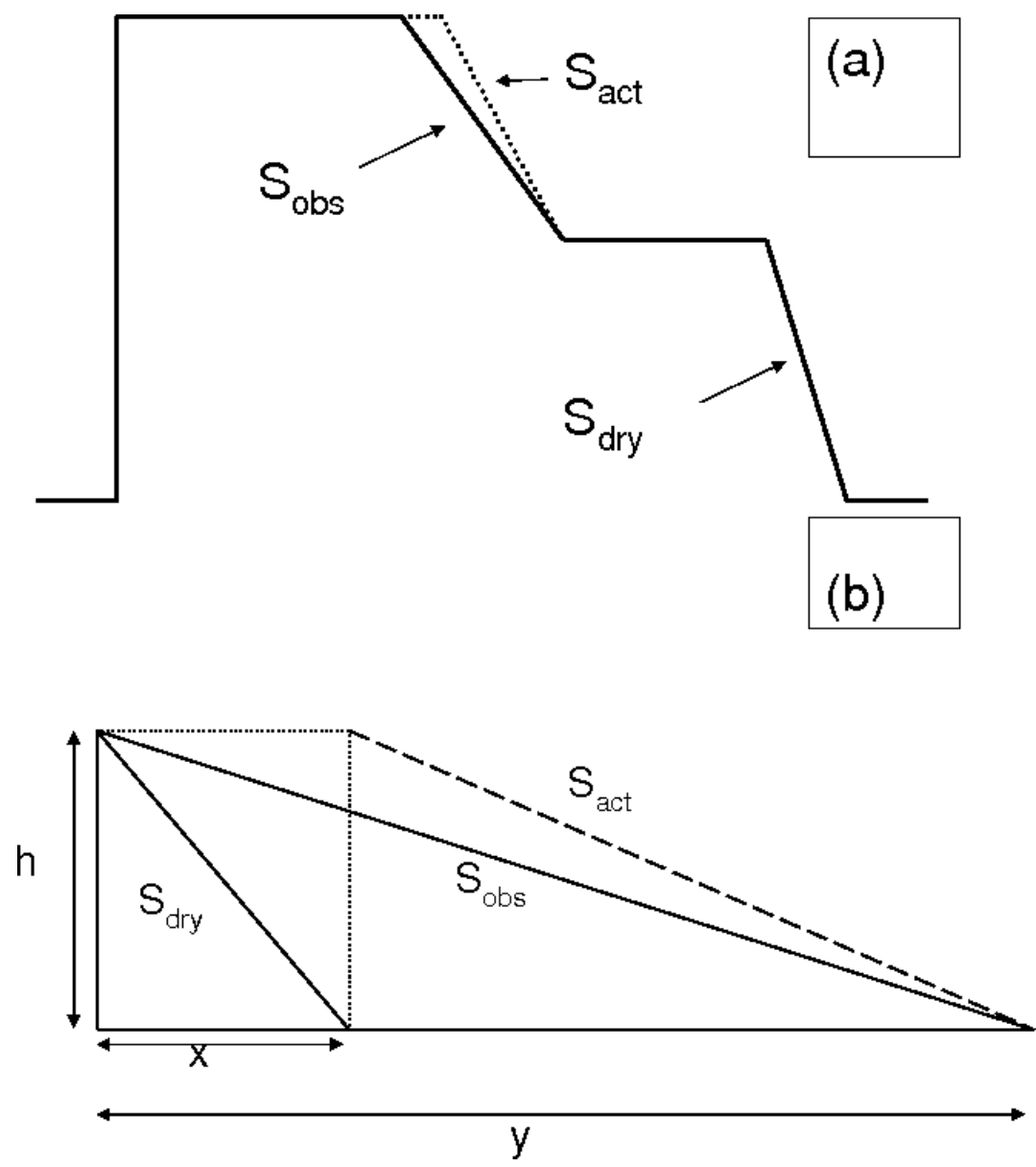

Fig. 4. (a) Illustration of the difference between observed and actual water concentration profiles obtained with magnetic resonance techniques. (b) Geometric construction to illustrate how the actual slope in the profiles is related to the observed slope.

of $P e$. Nevertheless, the experiments provide important information for those who wish to avoid non-uniform drying and accompanying skin formation in drying colloidal films. For instance, smaller particles (especially nanoparticles), thinner films, and slower evaporation rates (such as obtained in an atmosphere with a high relative humidity) will all favour uniform drying in the vertical direction.

\section{Acknowledgements}

This work was funded by the EC Framework 6 Integrated Project, NAnostructured waterborne POLymEr films with OutstaNding properties (NAPOLEON), under Contract No. IP 0118442. We thank Raquel Rodriguez (University of the Basque Country) for preparing the latex, Amanda Page (University of Surrey) for assistance with the manuscript preparation, and Alex Routh (University of Cambridge) for helpful discussions.

\section{References}

1. H. Jotischky, Surf. Coat. Intern. Pt. B Coatings Trans. 84 (2001) 11. 


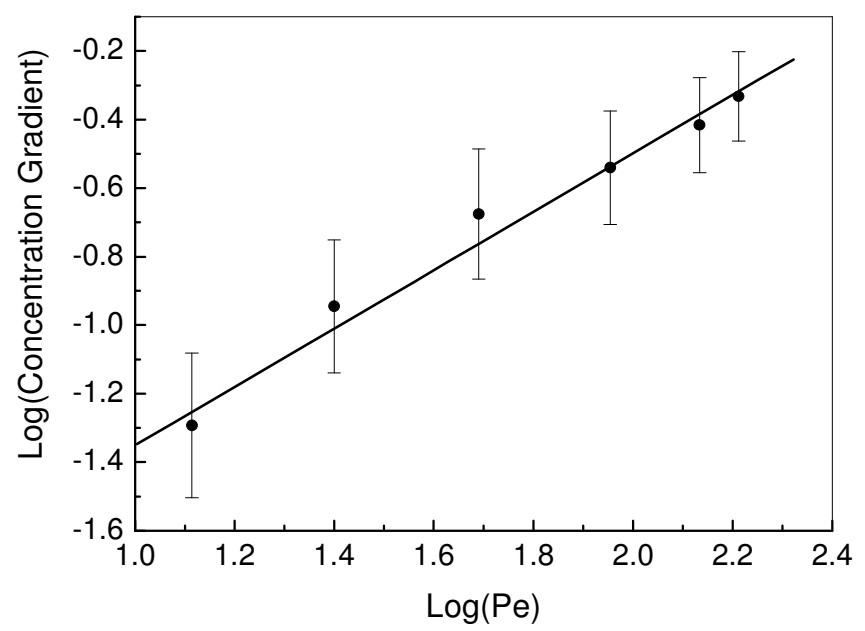

Fig. 5. Experimental test of the scaling prediction that $\mathrm{d} \phi_{w} / d z \sim P e^{1 / 2}$. The solid line is the best linear fit to the experimental data.

2. M.A. Winnik and J. Feng, J. Coat. Techn. 68 (1996) 39.

3. N. Agarwal and R.J. Farris, J. Appl. Polym. Sci. 72 (1999) 1407.

4. Y.K. Yang, H. Li and F. Wang, J. Adhes. Sci. Techn. 17 (2003) 1741.

5. A.J. Reuvers, Prog. Org. Coatings 35 (1999) 171.

6. A.F. Routh and W.B. Russel, Ind. Eng. Chem. Res. 40 (2001) 4302.

7. Q. Liao, L. Chen, X. Qu and X. Jin, J. Coll. Interf. Sci. 227 (2000) 84.

8. Y. Reyes and Y. Duda, Langmuir 21 (2005) 7057.

9. D. Guigner, C. Fischer and Y. Holl, Langmuir 17 (2001) 3598.

10. N. Dingenouts and M. Ballauff, Langmuir 15 (1999) 3283.

11. T. Narita, P. Hebraud and F. Lequeux, Eur. Phys. J. E 17 (2005) 69.

12. S. Erkselius, L. Wadso and O.J. Karlsson, J. Coll. Interf. Sci. 317 (2008) 83.

13. A.F. Routh, W.B. Russel, A.I.Ch.E. J. 44 (1998) 2088.

14. J.M. Salamanca, D.A. Faux, P.M. Glover, J.L. Keddie, P.J. McDonald, and A.F. Routh, Langmuir 17 (2001) 3202.

15. J. Mallegol, G. Bennett, P.J. McDonald, J.L. Keddie and O. Dupont, J. Adh. 82 (2006) 217.

16. D.P. Sheetz, J. Appl. Polym. Sci. 9 (1965) 3759.

17. V.R. Gundabala, W.B. Zimmerman and A.F. Routh, Langmuir 20 (2004) 8721.

18. A. F. Routh and W. B. Russel, Langmuir 15 (1999) 7762.

19. Y. Ma, H.T. Davis and L.E. Scriven, Prog. Org. Coat. 52 (2005) 46.

20. J. P. Gorce, D. Bovey, P. J. McDonald, P. Palasz, D. Taylor and J. L. Keddie, Eur. Phys. J. E 8 (2002) 421.

21. P.G. de Gennes, Eur. Phys. J. E 7 (2002) 31.

22. E. Ciampi and P.J. McDonald, Macromolecules 36 (2003) 8398.

23. A. F. Routh and W. B. Zimmerman, Chem. Eng. Sci. 59 (2004) 2961.

24. P. M. Glover, P. S. Aptaker, J. R. Bowler, E. Ciampi and P. J. McDonald, J. Mag. Res. 139 (1999) 90.

25. J.V. Sengers and J.T.R. Watson, J. Phys. Chem. Ref. Data 15 (1986) 1291.

26. P.J. McDonald and B. Newling, Rep. Prog. Phys. 61 (1998) 1441. 